\title{
Endoscopic Retrograde Cholangiopancreatography in Bilioenteric Anastomosis
}

\author{
Eun Taek Park \\ Division of Hepatobiliary and Pancreas, Department of Internal Medicine, Gospel Hospital, Kosin University College of Medicine, Busan, Korea
}

For diagnosis and treatment of pancreatobiliary diseases, endoscopic retrograde cholangiopancreatography (ERCP) is useful method nowadays and its technically success rate is usually in about $90 \%-95 \%$ of patients with normal gastric and pancreaticobiliary anatomy. Recently ERCP is significantly challenging after intestinal reconstruction, particularly in patients who have undergone pancreaticoduodenectomy (PD, classic Whipple's operation) or pylorus-preserving pancreatoduodenectomy (PPPD) with reconstruction. $\mathrm{PD}$ and $\mathrm{PPPD}$ relate to numerous techniques have been presented for reconstruction of the digestive tract and pancreaticobiliary tree during the resection bilioenteric stricture commonly occurs later in the postoperative course and developed in 5-year cumulative probability of biliary stricture rate of $8.2 \%$ and pancreaticoenteric stricture of $4.6 \%$. This complication was no difference in incidence between patients with benign or malignant disease. In PD or PPPD with reconstruction, short pancreatobiliary limb with biliojejunal anastomosis site is made usually, modestly success rate of intubation to blind loop and cannulation with conventional endoscope. However, in combined Reux-en-Y anastomosis, longer pancreatobiliary limb and additional Reux limb are obstacle to success intubation and cannulation by using conventional endoscope. In this situation, new designed enetroscope with dedicated accessories is efficient. Clin Endosc 2016;49:510-514

Key Words: ERCP; Pancreaticoduodenectomy; Pylorus-preserving pancreatoduodenectomy

\section{INTRODUCTION}

Currently, for diagnosis and treatment of pancreatobiliary diseases, endoscopic retrograde cholangiopancreatography (ERCP) is a useful method. Its technical success rate is about 90\%-95\% in patients with normal gastric and pancreaticobiliary anatomies. ${ }^{1}$ Recently, ERCP after intestinal reconstruction was found to be significantly challenging, particularly in patients who have undergone pancreaticoduodenectomy (PD; classic Whipples operation) or pylorus-preserving pancreatoduodenectomy (PPPD) with reconstruction. ${ }^{2}$

Received: September 20, 2016 Accepted: October 24, 2016

Correspondence: Eun Taek Park

Division of Hepatobiliary and Pancreas, Department of Internal Medicine, Gospel Hospital, Kosin University College of Medicine, 262 Gumcheon-ro, Seo-gu, Busan 49267, Korea

Tel: +82-51-990-6214, Fax: +82-51-990-5055, E-mail: euntpark@hanmail.net

(c) This is an Open Access article distributed under the terms of the Creative Commons Attribution Non-Commercial License (http://creativecommons.org/ licenses/by-nc/3.0) which permits unrestricted non-commercial use, distribution, and reproduction in any medium, provided the original work is properly cited.
Generally, PD and PPPD are performed for pancreatic and periampullary malignant diseases. However, recently, various benign diseases such as complicated or decompensated chronic pancreatitis, intraductal papillary mucinous neoplasm with malignant potential, and symptomatic mucinous cystic neoplasm have been included as indications, accounting for an estimated $9.2 \%$ of all PD and PPPD procedures performed. ${ }^{3}$

In high-volume centers, PD or PPPD is presently associated with a mortality rate of $<5 \%$ and an operation-related morbidity of $40 \%-50 \%$. The most frequent complications of PD or PPPD are pancreatic fistula, delayed gastric emptying, and hemorrhage. Less frequent complications include ischemic, infectious, and biliary complications, including bilioenteric stricture. $^{4}$

Bilioenteric stricture commonly occurs later in the postoperative course, with a 5 -year cumulative probability of $8.2 \%$ for biliary stricture and $4.6 \%$ for pancreaticoenteric stricture. Recurrent cases that require further treatment occur in about $20 \%-25 \%$ of cases. The incidence of this complication showed no significant difference between patients with benign or 
those with malignant disease. ${ }^{5}$

This review focuses on the technical challenges in performing ERCP in PD or PPPD with bilioenteric and pancreatoenteric anastomosis, and on strategies to overcome them.

\section{WHIPPLE'S OPERATION (PD) OR PPPD WITH INTESTINAL RECONSTRUCTION}

PD and PPPD have been performed in relation to numerous other techniques and have been introduced for reconstruction of the digestive tract and pancreaticobiliary tree during resection. The advantages of PPPD include long-term improvement in gastrointestinal function, as indicated by increased postoperative weight gain, fewer peptic ulcers, and less dumping. In addition, it simplifies the operation and thus leads to shorter operation time and less intraoperative blood loss than conventional PD. ${ }^{6}$

The PPPD technique needs at least three or four anastomoses, end-to-side pylorus-jejunostomy (only biliopancreatic limb) or end-to-side jejunojejunostomy (in Roux-en-Y reconstruction), end-to-side hepaticojejunostomy, and end-to-side or end-to-end pancreaticojejunostomy.

Especially end-to-side hepaticojejunostomy is a widely adapted technique for variable conditions such as liver transplantation, history of bile duct injuries or trauma, biliary strictures, or prior bilioenteric operations with stricture formation, choledochal cyst resection, recurrent biliary stones, hilar cholangiocarcinoma, and other biliary malignant tumors. ${ }^{7}$

Thorough knowledge and exact information on postoperative anatomy and training in conventional ERCP are needed before starting to perform ERCP procedures in patients with bilioenteric anastomosis. For example, magnetic resonance cholangiopancreatigraphy (MRCP) can be highly informative for understanding anatomies related to hepaticojejunostomy status.

Over 60 evolutional techniques have been introduced for reconstruction of the digestive and pancreaticobiliary tracts after PD or PPPD, including hepaticojejunostomy. ${ }^{8}$ In these techniques, the most important concept is that the anastomosis should be made only in a single limb of the bowel, that is, the afferent limb (loop), which is linked to the lesser curvature of the stomach to form the biliopancreatic limb. Consequently, the afferent jejunal loop is anastomosed to the common bile duct (hepaticojejunostomy); and the afferent jejunal loop, to the pancreatic remnant (pancreaticojejunostomy) in one limb of the bowel. Drainage for the biliary and pancreatic ductal systems is established via only one afferent limb.

The afferent loop (biliopancreatic limb) or Roux-en-Y (Roux limb) varies in length (shorter limbs, $<40 \mathrm{~cm}$ or longer limbs, $>60$ $\mathrm{cm}$ ) depending on the operative technique used in PD or PPPD.

\section{WHIPPLE'S OPERATION (PD) OR PPPD WITH ROUX-EN-Y ANASTOMOSIS}

Roux-en-Y reconstruction was developed at the end of the 19th century by the Swiss surgeon Cesar Roux (1857-1934). In Roux-en-Y reconstruction, long afferent limbs are frequently reconstructed in patients with morbid obesity, and hepaticojejunostomy is performed for living-donor liver transplantation (LDLT), PD for pancreatic adenocarcinoma and ampullary carcinoma, total gastrectomy for proximal stomach cancer, and choledochojejunostomy for stone or benign biliary disease. ${ }^{9}$

The number of Roux-en-Y reconstruction cases has increased as a result of the recent spread of laparoscopic surgery. In pancreatobiliary surgery (PD or PPPD), usually short-limb (pancreatobiliary and Roux limbs) Roux-en-Y reconstruction with bilioenteric/pancreatoenteric anastomosis is made. Hepaticojejunostomy with Roux-en-Y operation does not change the duodenum, making ERCP easy to perform for pancreatic disease. $^{10}$

In planning an ERCP for patients who are indicated to undergo Roux-en-Y reconstruction, the following considerations are needed: (1) Approximate length of the Roux-en-Y limb, which is determined in medical conditions such as a short limb in PD or PPPD and a long limb in bariatric surgery. (2) The Roux-en-Y, which has a short limb (approximately 40 $\mathrm{cm}$ ), may be found within the antecolic anastomosis, whereas the long limb (approximately $60 \mathrm{~cm}$ ) may be found within the retrocolic anastomosis. (3) Enteral anastomosis is usually performed with an end-to-side pattern, whereas combined Braun anastomosis is performed with a side-to-side pattern. ${ }^{11}$

\section{WHIPPLE'S OPERATION (PD) OR PPPD AND THE TECHNICAL LIMITATIONS OF ERCP}

ERCP has many technical limitations when performed with PD or PPPD with bilioenteric anastomosis (Fig. 1).

\section{Long afferent loop}

The total length of the afferent loop is dependent on the medical condition and operative opinion, and the location of the jejunal loop is related to the mesocolon. Prototype oblique-viewing duodenoscopy is available for PD or PPPD with or without Roux-en-Y anastomosis reconstruction. As mentioned earlier, PD or PPPD with enteral anastomosis is made with a short afferent loop ( $<40 \mathrm{~cm}$ length).

However, if the endoscope cannot reach the blind end, a forward-viewing gastroscope or baby colonoscope, with or 

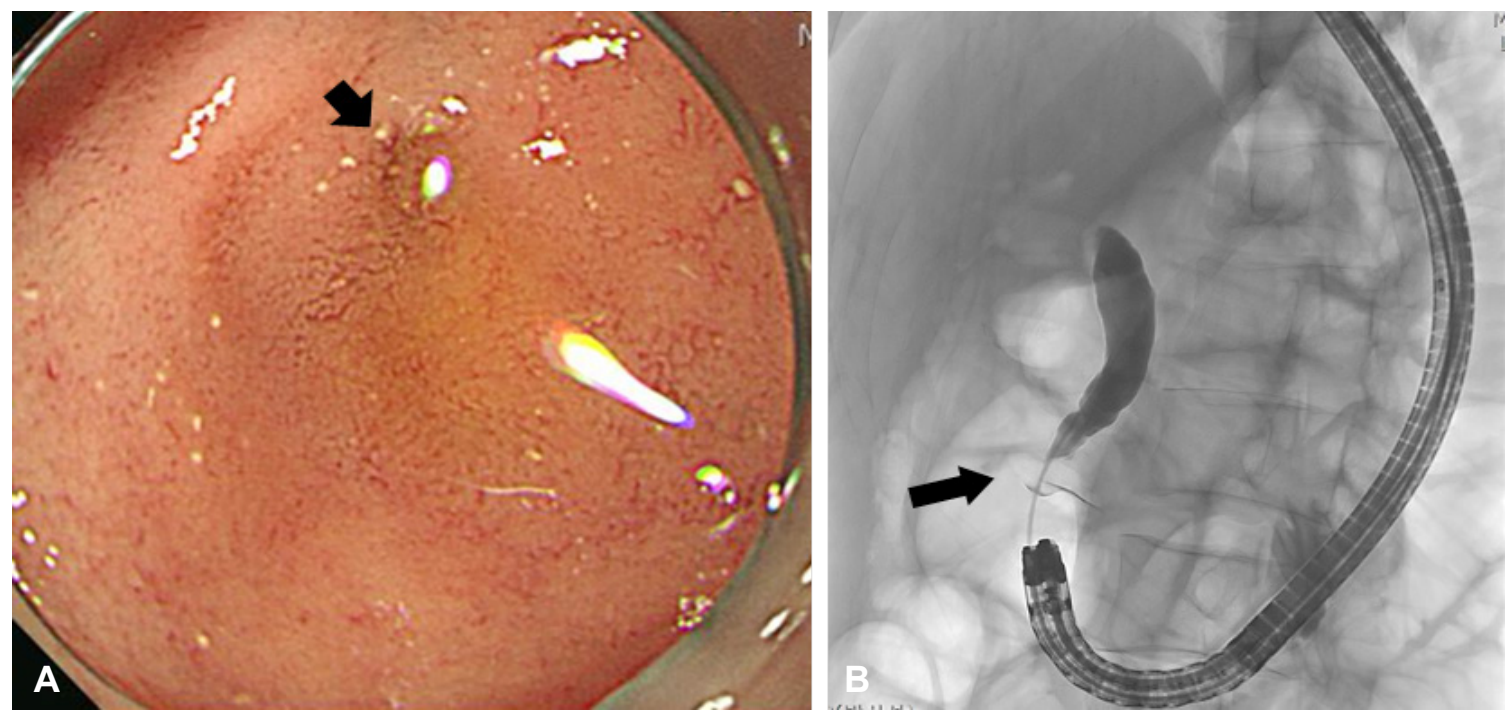

Fig. 1. Endoscopic retrograde cholangiopancreatography (ERCP) image obtained using a cap-assisted forward gastroscopy in a patient with pylorus-preserving pancreatoduodenectomy (PPPD) who underwent a hepaticojejunostomy. (A) Endoscopic image of the hepaticojejunal anastomotic stricture site (short arrow). (B) Fluoroscopy image of the reaching to create the hepatojejunal anastomosis (long arrow).

without an additional transparent distal cap, is used instead. The forward-viewing endoscope has better endoscopic orientation. Sometimes, device-assisted enteroscopy (DAE) is needed. ${ }^{12}$

\section{Angulation of the afferent loop}

Postoperative adhesion is an important cause of angulation. It is suspected during cleaning of regional lymphatics of malignant tumors. Loop formation in the remnant stomach is another cause of angulation. It is dependent on the length of the afferent loop. A long afferent limb related to Roux-en-Y may lead to abrupt angulation in the gastroenteral and jejunojejunal anastomosis sites in PD or PPPD.

If persistent loop formation with deep angulation occurs, abdominal compression or changing the patient's position is useful to release the loop formation and overcome angulation. Addition use of a colonoscope (variable stiffness), steerable tip of the colonoscope, and semi-rigid overtube DAE prevents loop formation near the gastric remnant. ${ }^{13}$

\section{Cannulation of bilioenteric or pancreatoenteric anastomosis}

In PD or PPPD, the hepaticojejunostomy orifice is made with an end-to-side anastomosis located about $10 \mathrm{~cm}$ distal to the blind end. Intermittent antiperistalsis with the presence of some bile juice can be observed on endoscopy. A pancreaticojejunostomy orifice is made with an end-to-end or endto-side anastomosis, with the pancreatic duct sutured to the jejunal mucosa (mucosa-to-mucosal anastomosis), or with a "dunking" anastomosis (the pancreatic stump is invaginated into the jejunum). On endoscopy, an anastomosis orifice can be observed depending on the transparency of the pancre- atic juice and its size. Hence, finding an anastomosis orifice is more difficult than finding a hepaticojejunostomy orifice. While cannulation can be used to create an orifice, the papilla orientation often changes. ${ }^{10}$

In PD or PPPD, because the bilioenteric or pancreatoenteric anastomosis has no intact papilla with the sphincter of Oddi, forming an orifice by using cannulation seems easier than forming a normal papilla orifice by using gastric bypass surgery.

\section{WHIPPLE'S OPERATION (PD) OR PPPD AND THE CHOICE OF THE MOST APPROPRIATE INSTRUMENT DURING ERCP}

In PD or PPPD, a short pancreatobiliary limb $(<40 \mathrm{~cm})$ with hepaticojejunal and pancreaticojejunal anastomoses is already reconstructed. Sometimes, Roux-en-Y reconstruction is additionally performed in the pancreatobiliary limb, in combination with the Roux limb created with jejunojejunal anastomosis. $^{14}$

In this situation, the choice of an adequate endoscope is made based on several factors. The first consideration is the length of the afferent limb. For PD or PPPD, the conventional duodenoscope or cap-assisted forward-viewing gastroscope can be used owing to the short afferent limb, but sometimes length limitation exists. The baby colonoscope or push enteroscope, which is longer, is an alternative tool. However, with these forward-viewing endoscopes without an elevator, cannulation is difficult in spite of successfully accessing the blind loop. In PD or PPPD with Roux-en-Y reconstruction, because 
the long Roux and pancreaticobiliary limbs are reconstructed, a deep-reaching enteroscope (double-balloon enteroscopy [DBE] or single-balloon enteroscopy [SBE]) is adequate..$^{15-17}$

The second consideration is how to overcome angulation. In PD or PPPD without Roux-en-Y anastomosis, deep angulation may occur in the gastrojejunal anastomosis site owing to adhesion. In this situation, cap assisted forward viewing endoscopy is safe and better overcome angulation. In PD or PPPD with Roux-en-Y anastomosis, from the start of the procedure, a deep-reaching enteroscope is useful for overcoming angulation. Gastrojejunostomy and jejunojejunostomy anastomosis often result in deep angulation and induce perforation. ${ }^{18}$

The third consideration is the instrument itself. The currently used endoscopes for performing ERCP are not primarily designed for this purpose (e.g., enteroscopes have been adapted for deep small bowel examination only). In PD or PPPD with Roux-en-Y reconstruction, owing to the length of the afferent limb, as mentioned earlier, the standard enteroscopes (DBE or SBE, with a $200-\mathrm{cm}$ working length and a working channel diameter of $2.8 \mathrm{~mm}$ ) are available. ${ }^{15,19-22}$ However, they limit the types of ERCP accessories that may be used because of the conventional devices for ERCP are too short to be used and complex procedures could not be performed. Thus, a short deep-reaching enteroscope (with a 152-cm working length and working channel diameter of $3.2 \mathrm{~mm}$ ) is developed, allowing the use of standard ERCP accessories. ${ }^{21,23-28}$

\section{WHIPPLE'S OPERATION (PD) OR PPPD WITH COMPLICATIONS OCCURRING DURING ERCP}

The overall complication risk ranges from $0 \%$ to $19.5 \%$ in ERCP procedures for patients with altered anatomies. In PD or PPPD, deep angulation is established by using a reconstructed intestinal anastomosis. Perforation is most serious and frequent develops as a post-ERCP complication. The perforation site is dependent on the intestinal reconstruction method used.

In PD or PPPD with Roux-en-Y, the gastrojejunal and jejunojejunal anastomosis site is vulnerable. Sometimes, a fixed and torqued afferent loop is also perforated during the procedure. Less frequent complications include bleeding, cholangitis, mucosal tears, and post-ERCP pancreatitis. ${ }^{9-11}$

\section{CONCLUSION}

ERCP is technically challenging in patients with a history of operation and intestinal reconstruction. In PD or PPPD with reconstruction, a short pancreaticobiliary limb is usually made in the biliojejunal anastomosis site, with a modest success rate of intubation to the blind loop and cannulation with a conventional endoscope.

However, in combined Roux-en- $Y$ anastomosis, a longer pancreatobiliary limb and additional Roux limb are obstacles to the success of intubation and cannulation by using a conventional endoscope. In this situation, a newly designed enteroscope with dedicated accessories would be efficient.

\section{Conflicts of Interest}

The author has no financial conflicts of interest.

\section{REFERENCES}

1. Chahal P, Baron TH, Topazian MD, Petersen BT, Levy MJ, Gostout CJ. Endoscopic retrograde cholangiopancreatography in post-Whipple patients. Endoscopy 2006;38:1241-1245.

2. Park BK, Jeon TJ, Jayaraman V, et al. Endoscopic Retrograde Cholangiopancreatography in Patients with Previous Pancreaticoduodenectomy: A Single-Center Experience. Dig Dis Sci 2016;61:293-302.

3. Reid-Lombardo KM, Ramos-De la Medina A, Thomsen K, Harmsen WS, Farnell MB. Long-term anastomotic complications after pancreaticoduodenectomy for benign diseases. J Gastrointest Surg 2007;11:17041711.

4. House MG, Cameron JL, Schulick RD, et al. Incidence and outcome of biliary strictures after pancreaticoduodenectomy. Ann Surg 2006;243:571-576; discussion 576-578.

5. Demirjian AN, Kent TS, Callery MP, Vollmer CM. The inconsistent nature of symptomatic pancreatico-jejunostomy anastomotic strictures. HPB (Oxford) 2010;12:482-487.

6. Balcom JH 4th, Rattner DW, Warshaw AL, Chang Y, Fernandez-del Castillo C. Ten-year experience with 733 pancreatic resections: changing indications, older patients, and decreasing length of hospitalization. Arch Surg 2001;136:391-398.

7. Amer S, Horsley-Silva JL, Menias CO, Pannala R. Endoscopic retrograde cholangiopancreatography in patients with surgically altered gastrointestinal anatomy. Abdom Imaging 2015;40:2921-2931.

8. Piessen G, Triboulet JP, Mariette C. Reconstruction after gastrectomy: which technique is best? J Visc Surg 2010;147:e273-e283.

9. Moreels TG. Altered anatomy: enteroscopy and ERCP procedure. Best Pract Res Clin Gastroenterol 2012;26:347-357.

10. Moreels TG. ERCP in the patient with surgically altered anatomy. Curr Gastroenterol Rep 2013;15:343.

11. Moreels TG. Endoscopic retrograde cholangiopancreatography in patients with altered anatomy: How to deal with the challenges? World J Gastrointest Endosc 2014;6:345-351.

12. Lee A, Shah JN. Endoscopic approach to the bile duct in the patient with surgically altered anatomy. Gastrointest Endosc Clin N Am 2013;23:483-504.

13. Kikuyama M, Itoi T, Ota Y, et al. Therapeutic endoscopy for stenotic pancreatodigestive tract anastomosis after pancreatoduodenectomy (with videos). Gastrointest Endosc 2011;73:376-382.

14. Ke S, Ding XM, Gao J, et al. A prospective, randomized trial of Rouxen-Y reconstruction with isolated pancreatic drainage versus conventional loop reconstruction after pancreaticoduodenectomy. Surgery 2013;153:743-752.

15. Shimatani M, Takaoka M, Matsushita M, Okazaki K. Endoscopic approaches for pancreatobiliary diseases in patients with altered gastrointestinal anatomy. Dig Endosc 2014;26 Suppl 1:70-78. 
16. Ross AS. Endoscopic retrograde cholangiopancreatography in the surgically modified gastrointestinal tract. Gastrointest Endosc Clin N Am 2009;19:497-507.

17. Matsushita M, Takakuwa H, Uchida K, Nishio A, Okazaki K. Techniques to facilitate ERCP with a conventional endoscope in patients with previous pancreatoduodenectomy. Endoscopy 2009;41:902-906.

18. Farrell J, Carr-Locke D, Garrido T, Ruymann F, Shields S, Saltzman J. Endoscopic retrograde cholangiopancreatography after pancreaticoduodenectomy for benign and malignant disease: indications and technical outcomes. Endoscopy 2006;38:1246-1249.

19. Tsutsumi K, Kato H, Muro S, et al. ERCP using a short double-balloon enteroscope in patients with prior pancreatoduodenectomy: higher maneuverability supplied by the efferent-limb route. Surg Endosc 2015;29:1944-1951

20. Itokawa F, Itoi T, Ishii K, Sofuni A, Moriyasu F. Single- and double-balloon enteroscopy-assisted endoscopic retrograde cholangiopancreatography in patients with Roux-en-Y plus hepaticojejunostomy anastomosis and Whipple resection. Dig Endosc 2014;26 Suppl 2:136-143.

21. Mönkemuller K, Jovanovic I. Endoscopic and retrograde cholangiographic appearance of hepaticojejunostomy strictures: A practical classification. World J Gastrointest Endosc 2011;3:213-219.

22. Kinney TP, Li R, Gupta K, et al. Therapeutic pancreatic endoscopy after Whipple resection requires rendezvous access. Endoscopy 2009;41:898901
23. Park JH, Ye BD, Byeon JS, et al. Approaching pancreatic duct through pancreaticojejunostomy site with double ballon enteroscope in patients with Roux-en-Y anatomy. Hepatogastroenterology 2013;60:1753-1758.

24. Cho S, Kamalaporn P, Kandel G, Kortan P, Marcon N, May G. 'Short' double-balloon enteroscope endoscopic retrograde cholangiopancreatography in patients with a surgically altered upper gastrointestinal tract. Can J Gastroenterol 2011;25:615-619.

25. Shimatani M, Matsushita M, Takaoka M, et al. Effective "short" double-balloon enteroscope for diagnostic and therapeutic ERCP in patients with altered gastrointestinal anatomy: a large case series. Endoscopy 2009;41:849-854

26. Siddiqui AA, Chaaya A, Shelton C, et al. Utility of the short double-balloon enteroscope to perform pancreaticobiliary interventions in patients with surgically altered anatomy in a US multicenter study. Dig Dis Sci 2013;58:858-864

27. Shah RJ, Smolkin M, Yen R, et al. A multicenter, U.S. experience of single-balloon, double-balloon, and rotational overtube-assisted enteroscopy ERCP in patients with surgically altered pancreaticobiliary anatomy (with video). Gastrointest Endosc 2013;77:593-600.

28. De Koning M, Moreels TG. Comparison of double-balloon and single-balloon enteroscope for therapeutic endoscopic retrograde cholangiography after Roux-en-Y small bowel surgery. BMC Gastroenterol 2016;16:98. 